\title{
Differentiation of Parkinsonism-Predominant Multiple System Atrophy from Idiopathic Parkinson Disease Using 3T Susceptibility-Weighted MR Imaging, Focusing on Putaminal Change and Lesion Asymmetry
}

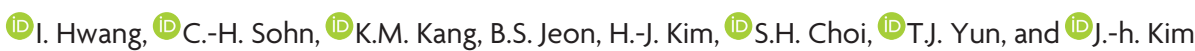

\begin{abstract}
BACKGROUND AND PURPOSE: Asymmetric presentation of clinical feature in parkinsonism is common, but correlatable radiologic feature is not clearly defined. Our aim was to evaluate 3 T susceptibility-weighted imaging findings for differentiating parkinsonismpredominant multiple system atrophy from idiopathic Parkinson disease, focusing on putaminal changes and lesion asymmetry.
\end{abstract}

MATERIALS AND METHODS: This retrospective cohort study included 27 patients with parkinsonism-predominant multiple system atrophy and 50 patients with idiopathic Parkinson disease diagnosed clinically. Twenty-seven age-matched subjects without evidence of movement disorders who underwent SWI were included as the control group. A consensus was reached by 2 radiologists who visually assessed SWI for the presence of putaminal atrophy and marked signal hypointensity on each side of the posterolateral putamen. We also quantitatively measured putaminal width and phase-shift values.

RESULTS: The mean disease duration was 4.7 years for the patients with parkinsonism-predominant multiple system atrophy and 7.8 years for the patients with idiopathic Parkinson disease. In the patients with parkinsonism-predominant multiple system atrophy, putaminal atrophy was frequently observed (14/27, 51.9\%) and was most commonly found in the unilateral putamen (13/14). Marked signal hypointensity was observed in 12 patients with parkinsonism-predominant multiple system atrophy (44.4\%). No patients with idiopathic Parkinson disease or healthy controls showed putaminal atrophy or marked signal hypointensity. Quantitatively measured putaminal width, phaseshift values, and the ratio of mean phase-shift values for the dominant and nondominant sides were significantly different between the parkinsonism-predominant multiple system atrophy group and the idiopathic Parkinson disease and healthy control groups $(P<.001)$.

CONCLUSIONS: 3T SWI can visualize putaminal atrophy and marked signal hypointensity in patients with parkinsonism-predominant multiple system atrophy with high specificity. Furthermore, it clearly demonstrates the dominant side of putaminal changes, which correlate with the contralateral symptomatic side of patients.

ABBREVIATIONS: IPD = idiopathic Parkinson disease; MSA-p = parkinsonism-predominant multiple system atrophy; MSA-c = cerebellar dysfunction type multiple system atrophy; ROC = receiver operating characteristic

$\mathbf{P}$ arkinsonism-predominant multiple system atrophy (MSA-p) is one of the Parkinson-plus syndromes that has a clinical manifestation similar to that of idiopathic Parkinson disease (IPD) and is often challenging to diagnose in its early stage. MR imaging plays a role in differentiating MSA-p from IPD and is included as an additional feature for the diagnosis of possible

Received December 16, 2014; accepted after revision April 23, 2015.

From the Departments of Radiology (I.H., C.-H.S., K.M.K, S.H.C., T.J.Y., J.-h.K.) and Neurology (B.S.J., H.-J.K.), Seoul National University Hospital, Seoul, Korea; Department of Radiology (C.-H.S.), Seoul National University College of Medicine, Seoul, Korea; and Institute of Radiation Medicine (C.-H.S.), Seoul National University Medical Research Center, Seoul, Korea.

Please address correspondence to Chul-Ho Sohn, MD, Department of Radiology, Seoul National University College of Medicine, 101 Daehak-ro, Jongno-gu, Seoul, 110-744, Korea; e-mail: neurorad63@gmail.com

三 Indicates article with supplemental on-line table.

http://dx.doi.org/10.3174/ajnr.A4442 multiple system atrophy. ${ }^{1}$ Various conventional and functional MR imaging findings regarding the putamen in MSA-p have been reported. ${ }^{2-6}$ However, these findings had limited sensitivity and specificity. ${ }^{6}$

An asymmetric presentation of clinical features is common for IPD in its early stage, while symmetric symptoms are more common in MSA-p than in IPD. ${ }^{7,8}$ However, the clinical manifestation of parkinsonism develops asymmetrically in many patients with MSA-p, and it has been reported that approximately $40 \%-$ $50 \%$ of patients with MSA-p present with initial asymmetric symptoms. ${ }^{8,9}$ This presentation increases the difficulty of clinically differentiating IPD from MSA-p in the early stage of disease. However, to our knowledge, there are few previous reports that used imaging to examine the asymmetry of putaminal abnormalities in MSA-p.

Susceptibility-weighted imaging (SWI), which was recently 
introduced and is now widely used in clinical brain imaging, reflects the physical magnetic properties of tissues because susceptibility changes in tissues, such as iron deposition, are very sensitive. ${ }^{10}$ In addition to the sensitivity of SWI to paramagnetic material, corrected phase images that are calculated to form final SWI can provide quantitative phase-shift values that reflect tissue iron content. ${ }^{11}$ Recently published studies attempted to use SWI to differentiate movement disorders, including MSA-p, ${ }^{12}$ and demonstrated different iron-deposition patterns between MSA-p and IPD by measuring phase-shift values by using corrected phase images of SWI sequences. ${ }^{13}$ However, most previous studies regarding SWI were performed on $1.5 \mathrm{~T}$ or weaker main magnetic field MR imaging machines. When main magnetic field is increased to 3T, spins process at a higher frequency, which may result in phase shifts caused by susceptibility changes being more exaggerated on SWI.

Thus, the purpose of the present study was to evaluate the imaging findings of 3T SWI for differentiating MSA-p from IPD, focusing on putaminal changes and lesion asymmetry.

\section{MATERIALS AND METHODS Study Population}

This retrospective cohort study was approved by the institutional review board of our hospital, and the requirement for informed consent was waived. The investigators searched all patients who were referred from the Movement Disorder Center to undergo brain MR imaging, including SWI, for further evaluation of parkinsonism between April 2010 and May 2012 ( $n=207)$. Among those patients, we enrolled subjects who had been initially, clinically diagnosed with either MSA-p $(n=33)$ or IPD $(n=50)$. All routine brain MR imaging protocols performed during the study period for the evaluation of movement disorders included SWI, except in 1 patient. Neurologists experienced with movement disorders (H.-J.K. and B.S.J.) made the final clinical diagnosis by reviewing the initial and follow-up clinical data in December 2013. MSA-p was diagnosed according to the "Second Consensus Statement on the Diagnosis of Multiple System Atrophy" ${ }^{1}$ and was categorized as probable or possible. IPD was diagnosed on the basis of the UK Parkinson's Disease Society Brain Bank criteria. ${ }^{14}$

Five initial patients with MSA-p were excluded for the following reasons: an old intracerebral hemorrhage involved the basal ganglia $(n=1)$, diagnoses were changed to cerebellar dysfunction type multiple system atrophy (MSA-c) $(n=3)$, and final diagnosis found no movement disorder $(n=1)$. One initial patient with IPD was excluded due to lack of SWI by a technical error. One initial patient with MSA-p had the final diagnosis changed to IPD. Finally, there were 27 patients with MSA-p and 50 patients with IPD. Twenty-seven age-matched subjects who met the following criteria were enrolled as the control group: 1) They underwent brain MR imaging with SWI between September 2012 and November 2012, 2) had no definite focal lesions in the brain parenchyma, and 3) had no clinical evidence of neurodegenerative diseases or movement disorders. The control group subjects were age-matched 1:1 with the subjects with MSA-p. Finally, our study included 27 patients with MSA-p (17 probable and 10 possible), 50 patients with IPD, and 27 healthy control subjects.

\section{MR Imaging Protocol}

All subjects underwent brain MR imaging by using a 3T scanner (Magnetom Verio; Siemens, Erlangen, Germany) with a 32-channel head coil. A set of images was acquired by using a 3D fully flow-compensated gradient-echo SWI sequence along the transverse plane parallel to the anterior/posterior commissure lines. The imaging parameters were as follows: $\mathrm{TR}=28 \mathrm{~ms}, \mathrm{TE}=20$ $\mathrm{ms}$, flip angle $=15^{\circ}$, section thickness $=2 \mathrm{~mm}, \mathrm{FOV}=178 \times 220$ $\mathrm{mm}$, matrix size $=364 \times 448$, and number of excitations $=1$. Therefore, the voxel size was $0.49 \times 0.49 \times 2.0 \mathrm{~mm}^{3}$. The magnitude and phase images were processed to create final SWI on the MR imaging console workstation (syngo MR B17; Siemens). The corrected phase images and final processed SWI were used for further analyses.

\section{Image Interpretation}

Two radiologists (I.H. and C.-H.S., with 4 and 17 years of experience in neuroradiology, respectively), who reached a consensus, reviewed the processed SWI. The investigators qualitatively evaluated the imaging findings for putaminal atrophy and signal intensity of the posterolateral putamen compared with adjacent structures in the basal ganglia (eg, globus pallidus). In particular, loss of lateral convexity of the posterolateral putaminal border was also indicative of putaminal atrophy. ${ }^{2}$ In addition to the SWI findings, cerebellar and brain stem findings were also reviewed with T2-weighted axial images for the following: 1) hot-cross bun sign of the pons, 2) high signal intensity in the middle cerebellar peduncle, and 3) cerebellar atrophy. The putaminal signal intensity was graded by using a relative 4-step scale introduced by Kraft et $\mathrm{al},{ }^{15}$ in which the signal of the putamen was rated as higher $=0$, equal $=1$, hypointense $=2$, or markedly hypointense $=3$. Other image findings were rated as negative $=0$, suspicious $=1$, or definitely positive $=2$. Laterality was also recorded if a finding of putaminal atrophy was rated as suspicious or definitely positive or the signal intensity was rated as marked hypointensity. During the interpretation, images were presented randomly and investigators were blinded to the patients' diagnoses.

\section{Quantitative Image Analysis}

We quantitatively measured the putaminal width and phase-shift values in corrected phase images. Image analyses were also performed by 2 radiologists who performed qualitative image interpretation and reached a consensus. The most representative section demonstrating the largest width and most well-demarcated border of the posterior putamen was selected for further analysis. The images were analyzed by using ImageJ $1.46 \mathrm{r}$ software (National Institutes of Health, Bethesda, Maryland) with 400\% magnification to more easily determine the boundaries of the basal ganglia structures. Investigators drew 3 straight lines with widths of 3 pixels crossing the midportion, posterior half, and far posterior portion of the putamen in the right-to-left direction, as shown in Fig $1 A$. Using the Plot Profile function in ImageJ, we plotted phase-shift values along the straight line, and putaminal positive phase-shift values were easily recognized (Fig $1 A$ ). The width and the mean phase-shift value on each side of the putamen were recorded by using the 3 lines. The phase-shift value was recorded in Siemens Phase Units, which range from 0 to 4096 

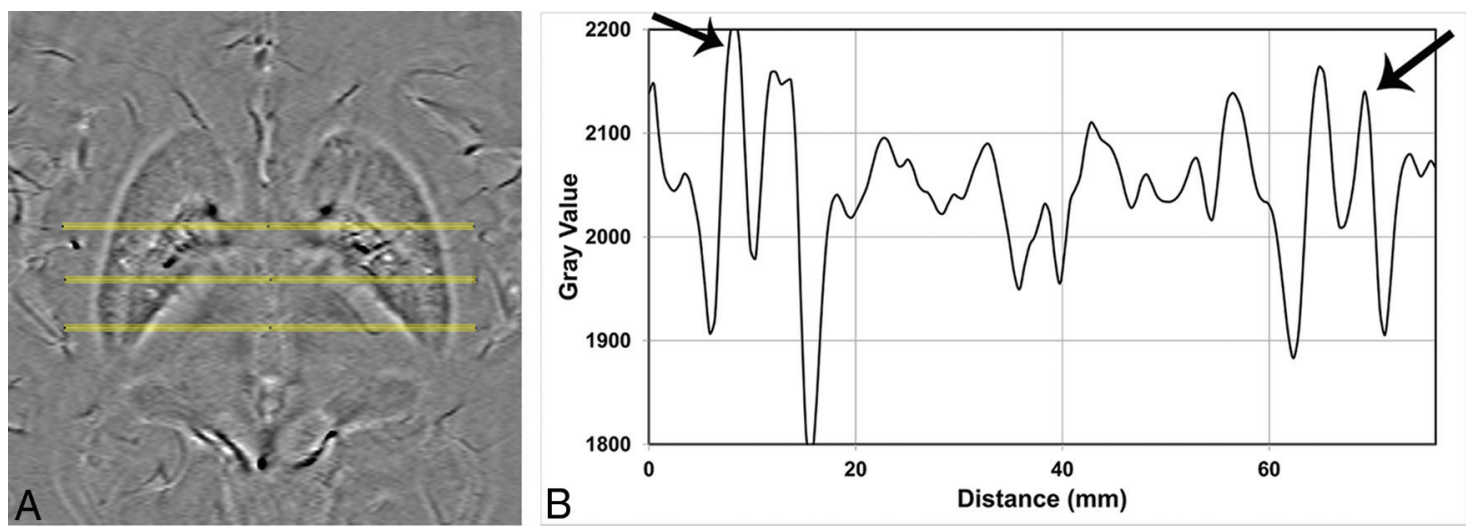

FIG 1. Measurement of putaminal width and mean phase-shift values from corrected phase images by using ImageJ software. $A$, Three lines crossing the mid-, posterior half, and far posterior putamen are drawn to allow measurements. $B$, The corresponding plot profile of the line crossing the far posterior portion of the putamen demonstrates increased phase-shift values in both putamina (arrows).

Table 1: Demographic and clinical characteristics of the study groups ${ }^{a}$

\begin{tabular}{|c|c|c|c|c|}
\hline & MSA-p & IPD & Healthy Control & $P$ Value \\
\hline No. & 27 & 50 & 27 & \\
\hline Mean age (yr) & $64.8 \pm 8.6(47-77)$ & $66.5 \pm 6.7(54-81)$ & $64.7 \pm 8.5(47-77)$ & $.521^{\mathrm{b}}$ \\
\hline Male/female ratio & 13:14 & 23:27 & $11: 16$ & $.850^{\mathrm{c}}$ \\
\hline Mean disease duration (yr) & $4.7 \pm 2.9$ & $7.8 \pm 5.0$ & NA & $.010^{\mathrm{d}}$ \\
\hline H\&Y scale & $2.85 \pm 0.72$ & $1.95 \pm 0.57$ & NA & $<.001^{\mathrm{d}}$ \\
\hline No. of patients with asymmetric presentation & 17 & 10 & NA & $<.001^{\mathrm{c}}$ \\
\hline
\end{tabular}

Note:-H\&Y scale indicates modified Hoehn and Yahr scale; NA, not applicable.

${ }^{a}$ Data are presented as mean $\pm S D$, with ranges in the parentheses.

${ }^{b}$ One-way analysis of variance test.

${ }^{c} \chi^{2}$ test.

${ }^{\mathrm{d}}$ Mann-Whitney $U$ test.

corresponding to $-\pi$ to $+\pi$ radian. ${ }^{16}$ To normalize the putamen width by brain size, we also measured the largest biparietal diameter of the brain parenchyma on the same image plane. The normalized putaminal width (putaminal width/biparietal diameter $\times$ mean of biparietal diameter of all subjects) was calculated and used for the statistical analysis. Dominant-side values (shorter putamen width or higher mean phase-shift value) and the mean of the values of both sides were calculated and used for the statistical analysis. Finally, to evaluate the asymmetry of the measured values, we also calculated the dominant/nondominant side ratios of the measured values. All analyses were performed for each straight line drawn at the mid-, posterior half, and far posterior portion of the putamen; then, the level that showed the most differentiation was selected and further analyzed.

\section{Neurologic Assessment}

Experienced neurologists (H.-J.K. and B.S.J.) assessed the final clinical diagnoses of IPD or MSA-p by reviewing the initial and follow-up clinical data. Those neurologists also assessed initial modified Hoehn and Yahr scale scores. In addition, the clinicalonset side of resting tremors or bradykinesia was recorded if the patient presented with asymmetric symptoms at the time of disease onset. If the symptom onset was not asymmetric or information regarding the side of onset was unclear, the more severe symptomatic side was recorded.

\section{Statistical Analyses}

All statistical analyses were performed by using MedCalc for Windows, Version 14.10.2 (MedCalc Software, Mariakerke, Belgium).
For all statistical analyses, a $P$ value $<.05$ was considered a statistically significant difference. For the qualitative assessment, the prevalence of each finding was calculated. In addition, the sensitivity and specificity for differentiating MSA-P from IPD were also investigated. To assess interrater agreement, we calculated the weighed $\kappa$ coefficient for 2 major qualitative imaging findings (putaminal atrophy and signal intensity of the posterolateral putamen) on the basis of the initial review data before the 2 reviewers arrived at a consensus. A $\chi^{2}$ test, Mann-Whitney test, 1-way analysis of variance test, and Kruskal-Wallis test were used to compare the clinicopathologic characteristics and quantitatively measured values for the MSA-p, IPD, and control subjects, as appropriate. A pair-wise comparison was used for the post hoc analysis. Furthermore, a receiver operating characteristic (ROC) curve was drawn for the variables that were significantly different by the KruskalWallis test for differentiating MSA-p from IPD. The area under the ROC curve was calculated to evaluate the diagnostic performance of those variables.

\section{RESULTS}

\section{Demographic and Clinical Characteristics}

The demographic and clinical characteristics of each group are described in Table 1. The mean disease duration at the time of MR imaging was significantly longer for the IPD group than the MSA-p group.

\section{Qualitative Image Interpretation}

Table 2 shows the prevalence of putaminal atrophy and marked signal hypointensity. No subject showed suspicious or definite 
Table 2: Prevalence of each image finding by visual interpretation of SWI in each group

\begin{tabular}{lccc}
\hline \multicolumn{1}{c}{ Imaging Finding } & MSA-p & Healthy Control \\
\hline No. & 27 & 50 & 27 \\
Putaminal atrophy & & & 0 \\
$\quad$ Suspicious & $3(11.1 \%)$ & 0 & 0 \\
$\quad$ Definite & $11(40.7 \%)$ & $24(48.0 \%)$ & $15(55.6 \%)$ \\
Putaminal signal intensity & $10(37.0 \%)$ & $22(44.0 \%)$ & $10(37.0 \%)$ \\
$\quad$ Hyperintense & $3(1.1 \%)$ & $2(7.4 \%)$ \\
Isointense & $2(7.4 \%)$ & 0 \\
Hypointense & $12(44.4 \%)$ & 0 & 0 \\
$\quad$ Markedly hypointense & $2(7.4 \%)$ & 0 & 0 \\
Hot-cross bun sign & $3(11.1 \%)$ & 0 & 0 \\
High signal intensity of middle cerebellar peduncle & $1(3.7 \%)$ & 0 & 0 \\
$\quad$ Suspicious & $11(40.7 \%)$ & $10(20.0 \%)$ & 0 \\
$\quad$ Definite & $3(11.1 \%)$ & 0 \\
Cerebellar atrophy & & \\
$\quad$ Suspicious & & \\
$\quad$ Definite & & \\
\hline
\end{tabular}
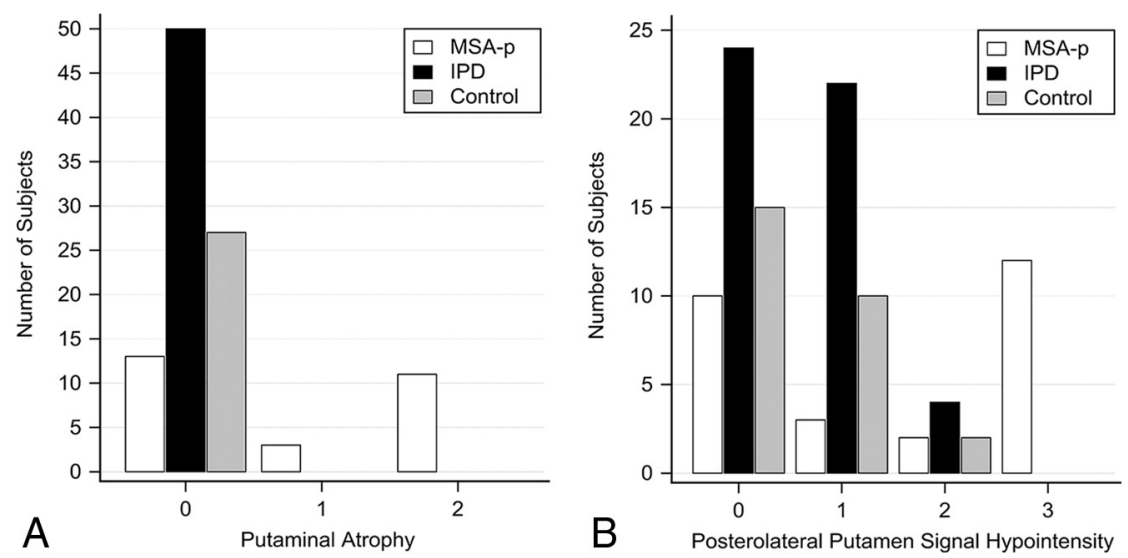

FIG 2. Distribution of putaminal atrophy $(A)$ and posterolateral putaminal signal intensity $(B)$ in each group. Putaminal atrophy: $0=$ negative, 1 = suspicious, 2 = definite; posterolateral putaminal signal intensity: 0 = hyperintense, $1=$ isointense, $2=$ hypointense, $3=$ markedly hypointense.

Table 3: Correlation between the clinically symptomatic side and asymmetry of imaging findings

\begin{tabular}{|c|c|c|c|c|c|c|}
\hline \multirow[b]{2}{*}{ Clinical Symptom } & \multicolumn{3}{|c|}{ Putaminal Atrophy $(n=14)$} & \multicolumn{3}{|c|}{ Marked Signal Hypointensity $(n=12)$} \\
\hline & Symmetric & Right & Left & Symmetric & Right & Left \\
\hline Symmetric & 0 & 1 & 2 & 1 & 1 & 0 \\
\hline Right-dominant & 1 & 0 & 4 & 2 & 0 & 3 \\
\hline Left-dominant & 0 & 5 & 1 & 1 & 3 & 1 \\
\hline
\end{tabular}

putaminal atrophy or marked signal hypointensity in the IPD or healthy control groups. Therefore, the specificity for each finding was $100 \%$. The sensitivities of putaminal atrophy and marked signal hypointensity were $51.9 \%(14 / 27)$ and $44.4 \%(12 / 27)$, respectively (Fig 2). Most of these findings were unilateral rather than bilateral (Table 3).

Table 3 summarizes the correlation between the clinically symptomatic side and asymmetry in the imaging findings. Among 14 subjects with MSA-p with suspicious or definite putaminal atrophy, 9 subjects presented with a symptomatic side that was contralateral to the atrophy side determined by imaging. The relationship between the clinically symptomatic side and marked signal hypointensity was weaker: Six subjects demonstrated a contralateral correlation between the symptomatic side and the marked hypointense signal side.

Simultaneous putaminal atrophy with marked putaminal signal hypointensity was observed in 11 of 27 patients with MSA-p (40.7\%). One patient with MSA-p had bilateral marked signal hypointensity without putaminal atrophy. Otherwise, all patients with MSA-p with marked signal hypointensity showed a loss of lateral convexity. Representative images from the patients with MSA-p and patients with IPD are shown in the Figs 3 and 4.

The weighted $\kappa$ coefficients for 2 major qualitative imaging findings - putaminal atrophy and signal intensity of the posterolateral putamen-were 0.728 (95\% CI, 0.561-0.894) and 0.636 (95\% CI, 0.461-0.812), respectively.

\section{Comparison of Measured Putaminal Width and Phase-Shift Values}

The On-line Table summarizes the measured values and the dominant/nondominant side ratios of the measured values at 

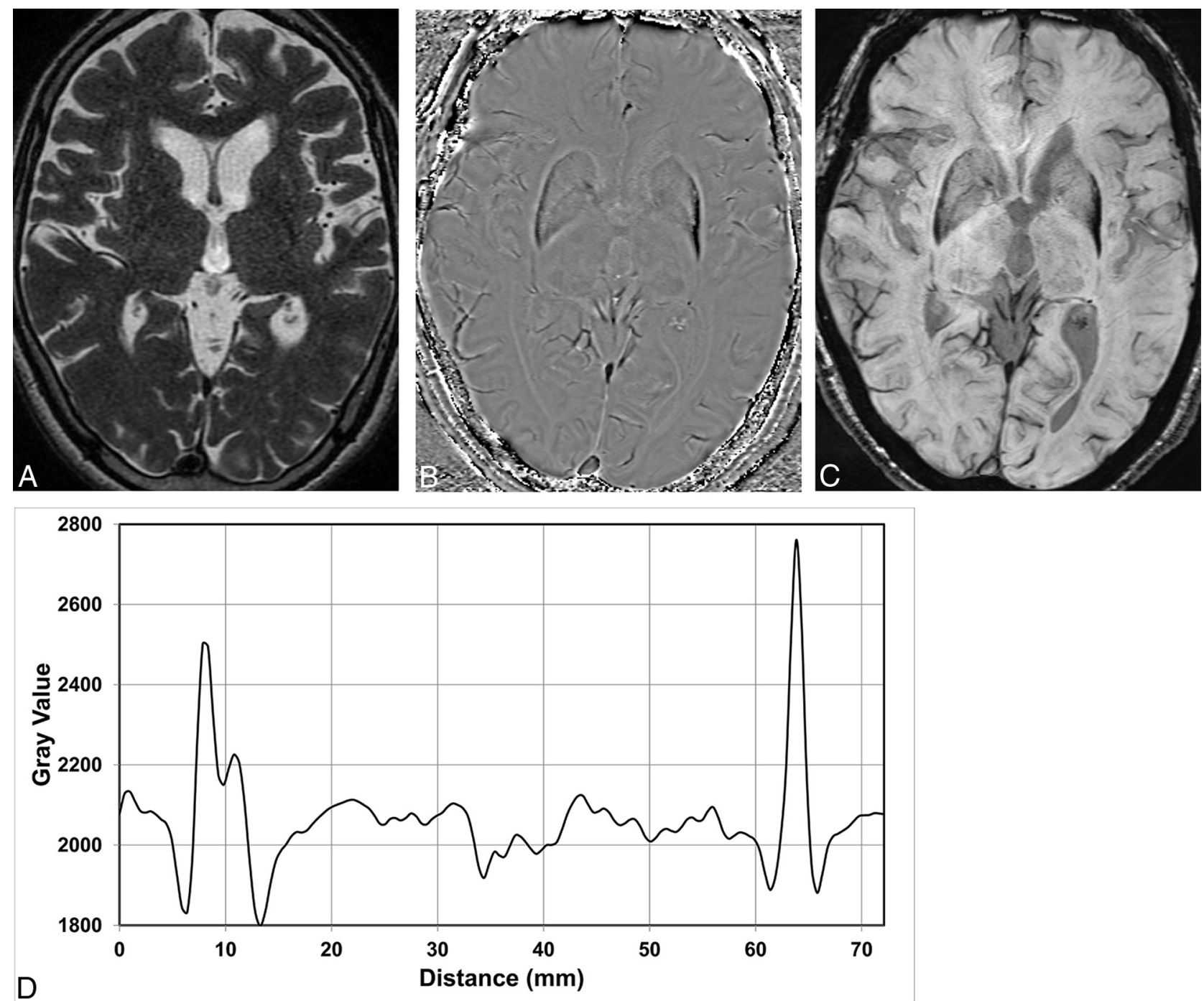

FIG 3. A 71-year-old woman who initially presented with right-leg dragging 3 years ago was clinically diagnosed as having probable MSA-p. A, On the T2-weighed axial image, the bilateral posterolateral putamen shows subtle hypointensity. The phase image $(B)$ and final SWI $(C)$ show a marked phase shift in the left posterolateral putamen with loss of lateral convexity of the posterolateral aspect of the putamen, suggesting atrophic change. $D$, Phase values along the far posterior of both putamina show asymmetric phase-shift values, and mean phase-shift values of the left putamina are measured as 2686.5 Siemens Phase Units.

the midportion, posterior half, and far posterior portion of the putamen. The measured values at the far posterior putamen showed the most significant differences between the MSA-p and IPD and control groups. Table 4 demonstrates the values of the far posterior putamen, and further analyses were based on the values measured at the far posterior putamen. The dominant-side (shorter) putaminal width and the mean of both putaminal widths were smaller in the MSA-p group compared with IPD and healthy control groups. The dominant-side mean phase-shift values and the mean phase-shift values of both putamina were also significantly different in the MSA-p group compared with the IPD and healthy control groups. The MSA-p group showed the highest phase-shift values among all groups. In terms of the dominant/nondominant side ratios, the differences in the putaminal width ratios were marginally insignificant between groups. The dominant/nondominant side ratio of the mean phase-shift values was significantly different between the MSA-p group and the IPD and healthy control groups; this difference suggested that the phase-shift value was significantly more asymmetric in the MSA-p group compared with the IPD and healthy control groups.

\section{ROC Curve Analysis for Values Measured to Differentiate MSA-p from IPD}

An ROC curve analysis was performed for the values that were quantitatively measured at the far posterior portion of the putamen to differentiate MSA-p from IPD. The area under the ROC curve was highest for the phase-shift value of both putamina $(0.803 ; 95 \%$ CI, $0.697-0.885)$, followed by the phase-shift value of the dominant side $(0.793 ; 95 \% \mathrm{CI}, 0.685-0.877)$, the putaminal width of the dominant side $(0.761 ; 95 \% \mathrm{CI}, 0.650-0.851)$, the mean putaminal width of both sides $(0.754$; 95\% CI, $0.643-$ 0.845 ), and the dominant-to-nondominant side ratio of the phase-shift value $(0.752 ; 95 \%$ CI, $0.640-0.843)$. Figure 5 demonstrates the ROC curve of the measured values. The optimal cutoff point of the phase-shift value of both putamina was 2121.6 Sie- 

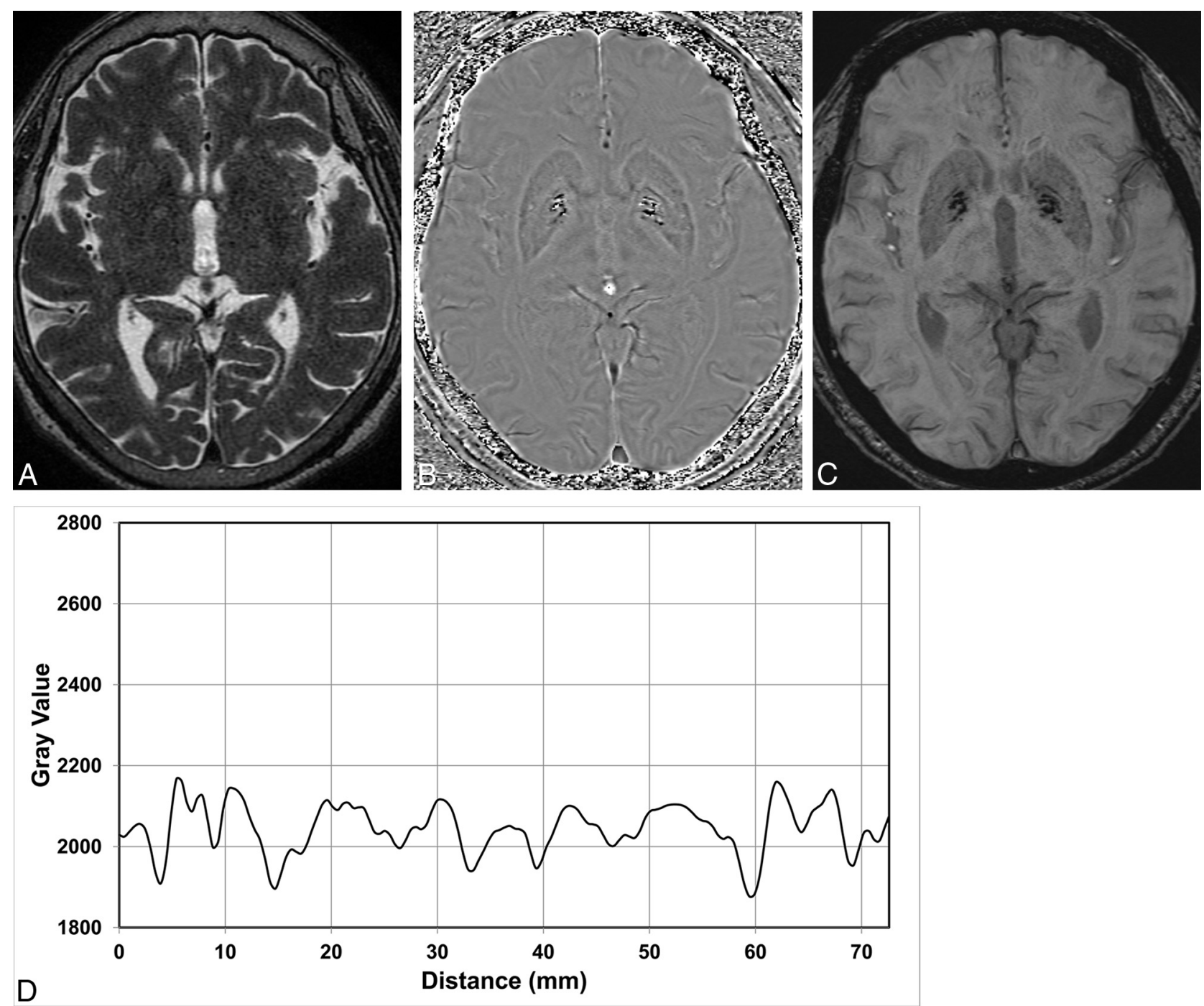

FIG 4. A 69-year-old woman who initially presented with gait disturbance and bradykinesia 8 years ago was clinically diagnosed with IPD. The T2-weighed axial image $(A)$, phase image $(B)$, and final SWI $(C)$ show no substantial signal alteration or atrophy in the bilateral putamina, while excessive iron deposition in the bilateral globus pallidus is observed. $D$, Phase values along the far posterior portion of both putamina show symmetric phase-shift values, and mean phase-shift values of the right and left putamina are measured as 2114.5 and 2111.7 Siemens Phase Units, respectively.

Table 4: Quantitatively measured putaminal width and phase-shift values: the ratios of dominant-to-nondominant-side values in the far posterior portion of putamen ${ }^{\mathrm{a}}$

\begin{tabular}{|c|c|c|c|c|}
\hline & MSA-p & IPD & Healthy Control & $P$ Value \\
\hline \multicolumn{5}{|l|}{ Measured values: dominant side } \\
\hline Putamen width ${ }^{b}$ & $3.17 \pm 0.65$ & $3.81 \pm 0.53$ & $3.87 \pm 0.47$ & $<.001$ \\
\hline Phase-shift value ${ }^{b}$ & $2322.2 \pm 236.9$ & $2121.5 \pm 44.4$ & $2136.7 \pm 45.8$ & $<.001$ \\
\hline \multicolumn{5}{|l|}{ Measured values: mean of both sides } \\
\hline Putamen width ${ }^{b}$ & $3.42 \pm 0.57$ & $3.98 \pm 0.52$ & $4.05 \pm 0.45$ & $<.001$ \\
\hline Phase-shift value ${ }^{b}$ & $2258.4 \pm 180.1$ & $2108.1 \pm 39.8$ & $2122.5 \pm 37.6$ & $<.001$ \\
\hline \multicolumn{5}{|l|}{ Ratio of dominant/nondominant side } \\
\hline Putamen width (shorter/longer side) & $0.866 \pm 0.123$ & $0.921 \pm 0.070$ & $0.912 \pm 0.048$ & .095 \\
\hline Phase-shift value ${ }^{b}$ (higher/lower side) & $1.057 \pm 0.065$ & $1.013 \pm 0.011$ & $1.013 \pm 0.011$ & $<.001$ \\
\hline
\end{tabular}

${ }^{\text {a }}$ Data are presented as means.

${ }^{b}$ MSA-p was significantly different $(P<.05)$ from IPD and healthy controls by post hoc analysis.

mens Phase Units, and the diagnostic performance was defined by $77.8 \%$ sensitivity and $76.0 \%$ specificity.

\section{DISCUSSION}

We investigated the 2 imaging findings of the putamen, putaminal atrophy and marked signal hypointensity, as a result of iron deposition on 3T SWI. Our results suggest that these image findings are very specific for patients with MSA-p (100\% specificity) and appear asymmetrically in those patients. The sensitivity of previously reported imaging findings for MSA-p ranged from approximately $50 \%$ to $85 \% .{ }^{6}$ Previous studies were most commonly performed by using an MR imaging system with a main magnetic 


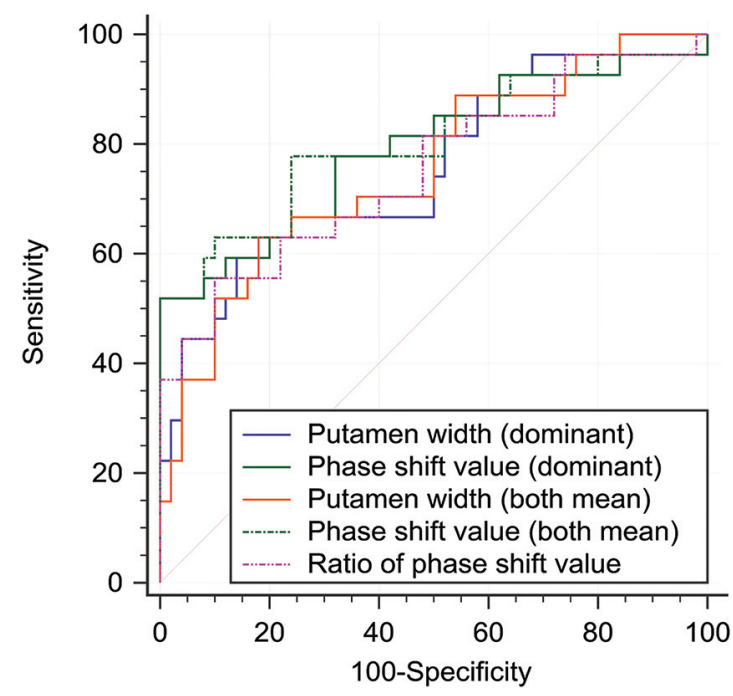

FIG 5. Receiver operating characteristic curves of values measured at the far posterior putamen to distinguish MSA-p from IPD.

field of $\leq 1.5 \mathrm{~T}$ and fast spin-echo techniques; this procedure may be the reason for the lower sensitivity of these studies because multiple refocusing radiofrequency pulses during fast spin-echo imaging reduce the paramagnetic effect of $\mathrm{T} 2$ hypointensity generated by iron deposition. ${ }^{3}$ However, our study was performed on a 3 T MR imaging scanner and also revealed only slightly $>50 \%$ sensitivity for the detection of MSA-p. In contrast, the specificity of our findings was $100 \%$, suggesting that putaminal atrophy and marked signal hypointensity in the posterior putamen could be specific, rather than sensitive, findings for MSA-p. The sensitivity of this study was similar or slightly lower than those previously published for studies that used conventional fast spin-echo-based 3T MR imaging findings. ${ }^{17,18}$ The sensitivity of our study was comparable with those of published studies that used $\mathrm{T}^{\star}{ }^{\star}$ weighted imaging. ${ }^{19,20}$ By ROC curve analysis of the quantitative phase-shift values, sensitivity was increased to $77.8 \%$, while specificity was slightly decreased (76.0\%). We suggest that quantitative measurement could be used to increase the sensitivity for the detection of MSA-p.

By visual assessment, putaminal atrophy and marked signal hypointensity usually occurred asymmetrically. Quantitative measurement of the phase-shift value of the posterior aspect of the putamen showed significantly higher asymmetry in the MSA-p group compared with the IPD and healthy control groups. These findings suggest that iron deposition in the posterior putamen in patients with MSA-p usually occurs asymmetrically. Furthermore, these imaging findings were frequently detected in the contralateral side of the symptomatic, dominant side. Our results were consistent with a few previous studies that used MR imaging and $\left[{ }^{18} \mathrm{~F}\right]$ fluorodeoxyglucose positron-emission tomography. ${ }^{21,22}$ As mentioned earlier, the clinical manifestation of parkinsonism develops asymmetrically in approximately 40\%$50 \%$ of patients with MSA-p. ${ }^{8,9}$ We believe that 3 T SWI can visualize asymmetric putaminal changes, including atrophy and iron deposition, in the contralateral, symptomatic side. This finding was not clearly indicated by previous imaging studies, even those that used 3 T imaging or 3T SWI. ${ }^{18,23}$

Our quantitative measurement method was not a volumetric method and did not include all putaminal areas or consider the high-iron-deposition area that was proposed to be meaningful by previous publications. ${ }^{11,13}$ Haacke et $\mathrm{al}^{11}$ demonstrated that putative iron deposition in the high-iron-content region increases with age. Our study was focused on imaging findings, and quantitative measurement was performed to support our imaging findings. For routine clinical practice, volume measurements would not be practical because they require meticulous drawing of the volume of interest and are time-consuming. In contrast, our measurement method can be easily performed, is less timeconsuming, and might be used for cases that are highly suspicious for MSA-p clinically but do not show asymmetry by visual analysis. When putaminal atrophy was severe, the posterior putamen was difficult to differentiate from the medial and lateral portions of the putamen and globus pallidus. This problem makes volumetric measurement of the posterior putaminal signal and volume separate from the pallidum difficult to achieve. However, our method can be easily used in this situation and could supplement the visual assessment of putaminal atrophy.

For differentiation of parkinsonism with imaging techniques, advanced MR imaging with a machining-learning technique showed excellent discrimination of IPD from atypical parkinsonism by support vector machine analysis by using DTI data. ${ }^{24}$ In that study, consecutive symptomatic patients with parkinsonism were referred for imaging to minimize confounding for individual-level classification. Our study also intended to differentiate IPD and MSA-p by using SWI for the patients who were referred for further evaluation of parkinsonism. Although machine learning-based classification showed excellent discrimination, that method could be easily but not directly available for routine clinical practice. Our visual inspection and measurement method of SWI can be directly applicable in routine clinical practice and does not require additional resources. In addition, our study demonstrated frequent asymmetric presentation of known putaminal change in MSA-p, which may not be discovered by support vector machine analysis.

In addition to the intrinsic limits of retrospective studies, a few limitations of this study should be mentioned. First, our study did not directly compare the diagnostic performance of our method with that of known findings on conventional spin-echo MR images and T1- and T2-weighted images. However, the diagnostic performance of our method could be compared with those of previous studies. Second, the longer disease duration in the IPD group may have decreased the proportion of unilateral disease manifestation. In the early disease stage, IPD frequently shows asymmetric symptoms, but in later stages, IPD has a more symmetric presentation. However, it is well-known that IPD shows no substantial putaminal changes by imaging studies, and our results were consistent with those of previous studies. ${ }^{6}$ Third, the diagnosis at the time of MR imaging did not discriminate between probable and possible MSA-p. Unfortunately, the data for probable and possible diagnosis for MSA-p were not available in all patients due to retrospective study design. The final diagnosis was made by reviewing the initial and follow-up clinical data in December 2013, while the imaging study had been done before May 2012. The direct comparison of imaging findings between final probable and possible MSA-p groups might lead to misclassifica- 
tion bias. Thus, imaging differences between patients with probable and possible MSA-p could not be analyzed.

\section{CONCLUSIONS}

3T SWI can visualize putaminal atrophy and marked signal hypointensity in patients with MSA-p with high specificity. Furthermore, it clearly demonstrates the dominant side of putaminal changes, which correlate with the contralateral symptomatic side of patients. We suggest that 3T SWI is a practical method for the evaluation of putaminal atrophy and iron deposition; thus, it could be helpful for differentiating MSA-p from IPD in clinical practice.

Disclosures: Beom S. Jeon—UNRELATED: Ipsen, Norvartis; Boehringer Ingelheim; the Korea Health 21 R\&D project; Ministry of Health \& Welfare, Republic of Korea; the National Research Foundation of Korea; Ministry of Education, Science and Technology; Advanced Biometric Research Center; Korean Science and Engineering Foundation; Seoul National University Hospital; the Mr. Chung Suk-Gyoo and Sinyang Cultural Foundation; and the Song Foundation, Comments: research support as Principal Investigator; Travel/Accommodations/Meeting Expenses Unrelated to Activities Listed: Korea Research-Based Pharmaceutical Industry Association and Korean Pharmaceutical Manufacturers Association. *Money paid to the institution.

\section{REFERENCES}

1. Gilman S, Wenning GK, Low PA, et al. Second consensus statement on the diagnosis of multiple system atrophy. Neurology 2008;71: 670-76 CrossRef Medline

2. Ito S, Shirai W, Hattori T. Evaluating posterolateral linearization of the putaminal margin with magnetic resonance imaging to diagnose the Parkinson variant of multiple system atrophy. Mov Disord 2007;22:578-81 CrossRef Medline

3. Watanabe $\mathrm{H}$, Ito $\mathrm{M}$, Fukatsu $\mathrm{H}$, et al. Putaminal magnetic resonance imaging features at various magnetic field strengths in multiple system atrophy. Mov Disord 2010;25:1916-23 CrossRef Medline

4. Tha KK, Terae S, Tsukahara A, et al. Hyperintense putaminal rim at 1.5 $\mathrm{T}$ : prevalence in normal subjects and distinguishing features from multiple system atrophy. BMC Neurol 2012;12:39 CrossRef Medline

5. Tsukamoto K, Matsusue E, Kanasaki Y, et al. Significance of apparent diffusion coefficient measurement for the differential diagnosis of multiple system atrophy, progressive supranuclear palsy, and Parkinson's disease: evaluation by 3.0-T MR imaging. Neuroradiology 2012;54:947-55 CrossRef Medline

6. Savoiardo M. Differential diagnosis of Parkinson's disease and atypical parkinsonian disorders by magnetic resonance imaging. Neurol Sci 2003;24(suppl 1):S35-37 CrossRef Medline

7. Hoehn MM, Yahr MD. Parkinsonism: onset, progression and mortality. Neurology 1967;17:427-42 CrossRef Medline

8. Gómez-Esteban JC, Tijero B, Ciordia R, et al. Factors influencing the symmetry of Parkinson's disease symptoms. Clin Neurol Neurosurg 2010;112:302-05 CrossRef Medline

9. Wüllner U, Schmitz-Hübsch T, Abele M, et al. Features of probable multiple system atrophy patients identified among 4770 patients with parkinsonism enrolled in the multicentre registry of the German Com- petence Network on Parkinson's disease. J Neural Transm 2007;114: 1161-65 CrossRef Medline

10. Haacke EM, Xu Y, Cheng YC, et al. Susceptibility weighted imaging (SWI). Magn Reson Med 2004;52:612-18 CrossRef Medline

11. Haacke EM, Miao Y, Liu M, et al. Correlation of putative iron content as represented by changes in $\mathrm{R} 2^{\star}$ and phase with age in deep gray matter of healthy adults. J Magn Reson Imaging 2010;32:561-76 CrossRef Medline

12. Gupta D, Saini J, Kesavadas C, et al. Utility of susceptibilityweighted MRI in differentiating Parkinson's disease and atypical parkinsonism. Neuroradiology 2010;52:1087-94 CrossRef Medline

13. Wang Y, Butros SR, Shuai X, et al. Different iron-deposition patterns of multiple system atrophy with predominant parkinsonism and idiopathetic Parkinson diseases demonstrated by phase-corrected susceptibility-weighted imaging. AJNR Am J Neuroradiol 2012;33:266-73 CrossRef Medline

14. Hughes AJ, Daniel SE, Kilford L, et al. Accuracy of clinical diagnosis of idiopathic Parkinson's disease: a clinico-pathological study of 100 cases. J Neurol Neurosurg Psychiatry 1992;55:181-84 CrossRef Medline

15. Kraft E, Trenkwalder C, Auer DP. T2*-weighted MRI differentiates multiple system atrophy from Parkinson's disease. Neurology 2002; 59:1265-67 CrossRef Medline

16. Haacke EM, Ayaz M, Khan A, et al. Establishing a baseline phase behavior in magnetic resonance imaging to determine normal vs. abnormal iron content in the brain. J Magn Reson Imaging 2007;26: 256-64 CrossRef Medline

17. Lee JY, Yun JY, Shin CW, et al. Putaminal abnormality on 3-T magnetic resonance imaging in early parkinsonism-predominant multiple system atrophy. J Neurol 2010;257:2065-70 CrossRef Medline

18. Feng JY, Huang B, Yang WQ, et al. The putaminal abnormalities on 3.0T magnetic resonance imaging: can they separate parkinsonismpredominant multiple system atrophy from Parkinson's disease? Acta Radiol 2015;56:322-28 CrossRef Medline

19. Arabia G, Morelli M, Paglionico S, et al. An magnetic resonance imaging $\mathrm{T} 2{ }^{\star}$-weighted sequence at short echo time to detect putaminal hypointensity in Parkinsonisms. Mov Disord 2010;25: 2728-34 CrossRef Medline

20. von Lewinski F, Werner C, Jörn T, et al. T2*-weighted MRI in diagnosis of multiple system atrophy: a practical approach for clinicians. J Neurol 2007;254:1184-88 CrossRef Medline

21. Kato T, Kume A, Ito K, et al. Asymmetrical FDG-PET and MRI findings of striatonigral system in multiple system atrophy with hemiparkinsonism. Radiat Med 1992;10:87-93 Medline

22. Kume A, Shiratori M, Takahashi A, et al. Hemi-parkinsonism in multiple system atrophy: a PET and MRI study. J Neurol Sci 1992; 110:37-45 CrossRef Medline

23. Meijer FJ, van Rumund A, Fasen BA, et al. Susceptibility-weighted imaging improves the diagnostic accuracy of $3 \mathrm{~T}$ brain MRI in the work-up of parkinsonism. AJNR Am J Neuroradiol 2015;36:454-60 CrossRef Medline

24. Haller S, Badoud S, Nguyen D, et al. Individual detection of patients with Parkinson disease using support vector machine analysis of diffusion tensor imaging data: initial results. AJNR Am J Neuroradiol 2012;33:2123-28 CrossRef Medline 